Honam Mathematical J. 35 (2013), No. 1, pp. 001-015

http://dx.doi.org/10.5831/HMJ.2013.35.1.1

\title{
SHEAF-THEORETIC APPROACH TO THE CONVOLUTION ALGEBRAS ON QUIVER VARIETIES
}

\author{
NAMHEE KWON
}

\begin{abstract}
In this paper, we study a sheaf-theoretic analysis of the convolution algebra on quiver varieties. As by-products, we reinterpret the results of $\mathrm{H}$. Nakajima. We also produce a refined form of the BBD decomposition theorem for quiver varieties. Finally, we study a construction of highest weight modules through constructible functions.
\end{abstract}

\section{Introduction}

Let $\mathfrak{g}$ be a symmetric Kac-Moody algebra, and let $\mathfrak{U}(\mathfrak{g})$ be the universal enveloping algebra of $\mathfrak{g}$. In $[9,10]$, the author introduced a new class of varieties which we call quiver varieties. These varieties are obtained from the representations of the framed quivers corresponding to the Cartan matrix of $\mathfrak{g}$. Nakajima used his quiver varieties to construct geometrically the finite dimensional irreducible representations of $\mathfrak{U}(\mathfrak{g})$ [10]. In Nakajima's geometric approach, the key ingredient was the construction of the algebra homomorphism

$$
\Phi: \mathfrak{U}(\mathfrak{g}) \longrightarrow \bigoplus_{\mathbf{v}^{1}, \mathbf{v}^{2}} H_{t o p}\left(Z\left(\mathbf{v}^{1}, \mathbf{v}^{2} ; \mathbf{w}\right)\right) .
$$

Here $Z\left(\mathbf{v}^{1}, \mathbf{v}^{2} ; \mathbf{w}\right)$ are certain Steinberg-type varieties which are obtained from the projective morphisms $\pi_{\mathbf{v}}: \mathfrak{M}(\mathbf{v}, \mathbf{w}) \longrightarrow \mathfrak{M}_{0}(\mathbf{v}, \mathbf{w})$ defined in $[10,3.18]$.

The homomorphism $\Phi$ is neither injective nor surjective in general. However, if we restrict our attention to a finite dimensional complex simple Lie algebra, then it turns out that the homomorphism $\Phi$ is surjective.

Received September 20, 2012. Accepted January 1, 2013.

2010 Mathematics Subject Classification. 17B10, 14L30, 16G20.

Key words and phrases. Convolution, Steinberg variety, quiver variety, highest weight module, Lie algebra, constructible function. 
This implies that any simple $\bigoplus_{\mathbf{v}^{1}, \mathbf{v}^{2}} H_{\text {top }}\left(Z\left(\mathbf{v}^{1}, \mathbf{v}^{2} ; \mathbf{w}\right)\right)$-module yields an irreducible representation of $\mathfrak{U}(\mathfrak{g})$.

For the underlying quivers of finite $A D E$ type, it is known from $[9$, Proposition 6.7] that the based variety $\mathfrak{M}_{0}(\mathbf{v}, \mathbf{w})$ of the projective morphism $\pi_{\mathbf{v}}$ has a wonderful stratification $\mathfrak{M}_{0}(\mathbf{v}, \mathbf{w})=\sqcup_{\mathbf{v}^{\prime} \leq \mathbf{v}} \mathfrak{M}_{0}^{r e g}\left(\mathbf{v}^{\prime}, \mathbf{w}\right)$, and the projective morphisms $\pi_{\mathbf{v}}$ become strictly semi-small (see [10, Corollary 10.11]). Hence, we can apply the Beilinson-Bernstein-Deligne decomposition theorem to the projective morphism $\pi: \sqcup_{\mathbf{v}} \mathfrak{M}(\mathbf{v}, \mathbf{w}) \longrightarrow$ $\cup_{\mathbf{v}} \mathfrak{M}_{0}(\mathbf{v}, \mathbf{w})$ whose restriction to each $\mathfrak{M}(\mathbf{v}, \mathbf{w})$ is $\pi_{\mathbf{v}}$. More explicitly, we obtain the following nice decomposition for the projective morphism $\pi$ :

$$
\pi_{*} \mathcal{C}=\bigoplus_{\varphi=\left(\mathfrak{M}_{0}^{r e g}\left(\mathbf{v}^{\varphi}, \mathbf{w}\right), \chi_{\varphi}\right)} L_{\varphi} \otimes I C_{\varphi},
$$

where $\mathcal{C}$ is the constant perverse sheaf on $\sqcup_{\mathbf{v}} \mathfrak{M}(\mathbf{v}, \mathbf{w})$ such that $\left.\mathcal{C}\right|_{\mathfrak{M}(\mathbf{v}, \mathbf{w})}$ $=\underline{\mathbb{C}}_{\mathfrak{M}(\mathbf{v}, \mathbf{w})}\left[\operatorname{dim}_{\mathbb{C}} \mathfrak{M}(\mathbf{v}, \mathbf{w})\right]$, and the direct sum is taken over the pairs $\varphi$ consisting of a stratum $\mathfrak{M}_{0}^{r e g}\left(\mathbf{v}^{\varphi}, \mathbf{w}\right)$ of $\mathfrak{M}_{0}(\mathbf{v}, \mathbf{w})$ and an irreducible local system $\chi_{\varphi}$ on $\mathfrak{M}_{0}^{\text {reg }}\left(\mathbf{v}^{\varphi}, \mathbf{w}\right)$. We point out that we have no shifts for the intersection cohomology complexes in the decomposition (1.1).

On the other hand, if we apply the sheaf-theoretic analysis to the convolution algebra $\bigoplus_{\mathbf{v}^{1}, \mathbf{v}^{2}} H_{\text {top }}\left(Z\left(\mathbf{v}^{1}, \mathbf{v}^{2} ; \mathbf{w}\right)\right)$ then we can show that

$$
\bigoplus_{\mathbf{v}^{1}, \mathbf{v}^{2}} H_{t o p}\left(Z\left(\mathbf{v}^{1}, \mathbf{v}^{2} ; \mathbf{w}\right)\right) \simeq \bigoplus_{\varphi} \operatorname{End}\left(L_{\varphi}\right),
$$

where $\left\{L_{\varphi}\right\}$ are the vector spaces that appear in (1.1) (see Theorem 4.2). Hence, the vector spaces $L_{\varphi}$ form a list of simple $\mathfrak{U}(\mathfrak{g})$-modules. This approach can be considered as a sheaf-theoretic version of Nakajima's theory that appeared in [10]. In particular, Equation (1.2) may be viewed as a sheaf-theoretic analogue of [10, Theorem 10.15].

It is well-known that the quiver varieties $\mathfrak{M}(\mathbf{v}, \mathbf{w})$ are connected [4]. By combining this fact with our sheaf-theoretic analysis of homology spaces over quiver varieties (see Theorem 4.1), we obtain a refined form of the decomposition (1.1). In more details, in this paper we obtain the following refined form of the decomposition (1.1):

$$
\pi_{*} \mathcal{C}=\underset{\varphi=\left(\mathfrak{M}_{0}^{r e g}\left(\mathbf{v}^{\varphi}, \mathbf{w}\right), \mathbb{C}_{\mathfrak{M}_{0}^{r e g}\left(\mathbf{v}^{\varphi}, \mathbf{w}\right)}\right)}{\bigoplus} L_{\varphi} \otimes I C_{\varphi},
$$

where $\underline{\mathbb{C}}_{\mathfrak{M}_{0}^{r e g}\left(\mathbf{v}^{\varphi}, \mathbf{w}\right)}$ is the constant local systems on each stratum $\mathfrak{M}_{0}^{\text {reg }}\left(\mathbf{v}^{\varphi}\right.$, $\mathbf{w})$, and the direct sum is taken over the pairs consisting of each stratum 
$\mathfrak{M}_{0}^{r e g}\left(\mathbf{v}^{\varphi}, \mathbf{w}\right)$ of $\mathfrak{M}_{0}(\mathbf{v}, \mathbf{w})$ and the locally constant system $\mathbb{C}_{\mathfrak{M}_{0}^{r e g}}\left(\mathbf{v}^{\varphi}, \mathbf{w}\right)$ on $\mathfrak{M}_{0}^{\text {reg }}\left(\mathbf{v}^{\varphi}, \mathbf{w}\right)$.

In the last section of this paper, we treat constructible functions to study the representation theory of $\mathfrak{U}(\mathfrak{g})$. In [9], Nakajima constructed irreducible representations of $\mathfrak{U}(\mathfrak{g})$ by using the vector space of constructible functions over the fiber $\pi^{-1}(0)$. However, in this paper we take an arbitrary fiber $\pi^{-1}(x)$ and construct irreducible representations of $\mathfrak{U}(\mathfrak{g})$ by using the constructible functions defined over the fiber $\pi^{-1}(x)$.

\section{Quiver varieties}

Let $\mathfrak{g}$ be a finite dimensional complex simple Lie algebra of type $A D E$, and let $(I, E)$ be the corresponding Dynkin diagram. Here $I$ and $E$ denote the sets of vertices and edges of the Dynkin diagram, respectively. Let $V=\left(V_{i}\right)_{i \in I}$ and $W=\left(W_{i}\right)_{i \in I}$ be collections of complex finite dimensional vector spaces attached to the set $I$. We write $\mathbf{v}$ and w for row vectors $\left(\operatorname{dim} V_{i}\right)_{i \in I}$ and $\left(\operatorname{dim} W_{i}\right)_{i \in I}$, respectively. Next, we denote by $H$ the set of pairs consisting of an edge and its orientation. For $h \in H$, we write out $(h)$ (resp., in $(h)$ ) for outgoing (resp., ingoing) vertex of $h$. For the above two collections $V$ and $W$ of vector spaces, we define the following vector space:

$$
\begin{aligned}
M(\mathbf{v}, \mathbf{w})= & \left(\bigoplus_{h \in H} \operatorname{Hom}\left(V_{\text {out }(h)}, V_{\text {in }(h)}\right)\right) \oplus\left(\bigoplus_{k \in I} \operatorname{Hom}\left(W_{k}, V_{k}\right)\right) \\
& \oplus\left(\bigoplus_{k \in I} \operatorname{Hom}\left(V_{k}, W_{k}\right)\right) .
\end{aligned}
$$

Each of three components of an element of $M(\mathbf{v}, \mathbf{w})$ will be denoted by $B=\left(B_{h}\right)_{h \in H}, i=\left(i_{k}\right)_{k \in I}$ and $j=\left(j_{k}\right)_{k \in I}$.

For $h \in H$, we write $\bar{h}$ to denote the same edge as $h$ with the reverse orientation. By the orientation of the Dynkin diagram $(I, E)$ we mean a subset $\Omega \subset H$ satisfying $\bar{\Omega} \cup \Omega=H$ and $\bar{\Omega} \cap \Omega=\emptyset$. For a fixed orientation $\Omega$ of $(I, E)$, we define a map $\epsilon: H \longrightarrow\{ \pm 1\}$ by

$$
\epsilon(h)= \begin{cases}1, & \text { if } h \in \Omega, \\ -1, & \text { if } h \in \bar{\Omega} .\end{cases}
$$

Define an action of $G_{\mathbf{v}}=\prod_{k \in I} G L\left(V_{k}\right)$ on $M(\mathbf{v}, \mathbf{w})$ by

$$
g \cdot(B, i, j)=\left(g B g^{-1}, g i, j g^{-1}\right),
$$


and let $\mu: M(\mathbf{v}, \mathbf{w}) \longrightarrow \bigoplus_{k \in I}$ End $\left(V_{k}\right)$ be a map whose $k$ th component is given by $\mu_{k}(B, i, j)=\sum_{i n(h)=k} \epsilon(h) B_{h} B_{\bar{h}}+i_{k} j_{k}$. Then, we define $\mathfrak{M}_{0}(\mathbf{v}, \mathbf{w})$ as the affine algebro-geometric quotient $\mu^{-1}(0) / / G_{\mathbf{v}}$. This is an affine variety, and its geometric points are exactly closed $G$-orbits on $\mu^{-1}(0)$.

On the other hand, it is known that we may have another quotient of $\mu^{-1}(0)$ through the geometric invariant theory quotient $[6,11]$. In more details, we first fix a character $\chi$ on $G_{\mathbf{v}}$ given by $\chi(g)=\prod_{k} \operatorname{det}\left(g_{k}^{-1}\right)$. Then, we define $\mathfrak{M}(\mathbf{v}, \mathbf{w})$ as the geometric invariant theory quotient $\mu^{-1}(0) / /{ }_{\chi} G_{\mathbf{v}}$ of $\mu^{-1}(0)$. We call this quotient a quiver variety. It is known that the quiver variety $\mathfrak{M}(\mathbf{v}, \mathbf{w})$ is a nonsingular quasi projective variety.

We say that an element $(B, i, j) \in \mu^{-1}(0)$ is stable if each subspace $S=\left(S_{k}\right)$ of $V=\left(V_{k}\right)$, which is invariant under $\left(B_{h}\right)$ (i.e., $B_{h}\left(S_{\text {out }(h)}\right) \subset$ $\left.S_{i n(h)}\right)$ and contained in $\operatorname{ker} j$ (i.e., $S_{k} \subset \operatorname{ker} j_{k}$ ), is actually equal to 0 . We denote by $\mu^{-1}(0)^{s}$ the set of all stable points in $\mu^{-1}(0)$. We recall that the geometric points of $\mathfrak{M}(\mathbf{v}, \mathbf{w})$ consists of $\mu^{-1}(0)^{s} / G$, that is $G$-orbit spaces on $\mu^{-1}(0)^{s}$.

If $(B, i, j) \in \mu^{-1}(0)$, then we denote by $[B, i, j]$ the corresponding geometric point in $\mathfrak{M}_{0}(\mathbf{v}, \mathbf{w})$. We will use the same notation $[B, i, j]$ to denote the geometric point of $\mathfrak{M}(\mathbf{v}, \mathbf{w})$ if $(B, i, j) \in \mu^{-1}(0)^{s}$.

Notice that we have a natural projective morphism

$$
\pi_{\mathbf{v}}: \mathfrak{M}(\mathbf{v}, \mathbf{w}) \longrightarrow \mathfrak{M}_{0}(\mathbf{v}, \mathbf{w})
$$

which sends an orbit $[B, i, j]$ to the unique closed orbit $\left[B_{0}, i_{0}, j_{0}\right]$ contained in $[B, i, j]$.

Let us now consider two quiver varieties $\mathfrak{M}\left(\mathbf{v}^{1}, \mathbf{w}\right)$ and $\mathfrak{M}\left(\mathbf{v}^{2}, \mathbf{w}\right)$. Then, we obtain projective morphisms $\pi_{\mathbf{v}^{i}}: \mathfrak{M}\left(\mathbf{v}^{i}, \mathbf{w}\right) \longrightarrow \mathfrak{M}_{0}\left(\mathbf{v}^{i}, \mathbf{w}\right)$ for $i=1$, 2. Since we have natural inclusions $\mathfrak{M}_{0}\left(\mathbf{v}^{i}, \mathbf{w}\right) \subset \mathfrak{M}_{0}\left(\mathbf{v}^{1}+\mathbf{v}^{2}\right.$, $\mathbf{w}$ ) for $i=1,2$, each $\pi_{\mathbf{v}^{i}}$ can be regarded as a morphism

$$
\pi_{\mathbf{v}^{i}}: \mathfrak{M}\left(\mathbf{v}^{i}, \mathbf{w}\right) \longrightarrow \mathfrak{M}_{0}\left(\mathbf{v}^{1}+\mathbf{v}^{2}, \mathbf{w}\right) .
$$

For the projective morphism $\pi_{\mathbf{v}}$ in $(2.1)$, we write $\mathfrak{M}(\mathbf{v}, \mathbf{w})_{x}$ for $\pi_{\mathbf{v}}^{-1}(x)$. In particular, we denote by $\mathfrak{L}(\mathbf{v}, \mathbf{w})$ the fiber $\pi_{\mathbf{v}}^{-1}(0)$.

For the affine varieties $\mathfrak{M}_{0}(\mathbf{v}, \mathbf{w})$ arising from the Dynkin diagrams of finite dimensional simple Lie algebras of type $A D E$, it is known that there exists a stratification

$$
\mathfrak{M}_{0}(\mathbf{v}, \mathbf{w})=\sqcup_{\mathbf{v}^{\prime} \leq \mathbf{v}} \mathfrak{M}_{0}^{r e g}\left(\mathbf{v}^{\prime}, \mathbf{w}\right),
$$


where $\mathfrak{M}_{0}^{\text {reg }}\left(\mathbf{v}^{\prime}, \mathbf{w}\right)$ is an open subset of $\mathfrak{M}_{0}\left(\mathbf{v}^{\prime}, \mathbf{w}\right)$ consisting of the points $[B, i, j]$ that have the trivial stabilizer in $G_{\mathbf{v}^{\prime}}$ (see $[10$, Lemma $3.27])$.

For a fixed $\mathbf{w}$, let us define the following projective morphism

$$
\pi: \sqcup_{\mathbf{v}} \mathfrak{M}(\mathbf{v}, \mathbf{w}) \longrightarrow \cup_{\mathbf{v}} \mathfrak{M}_{0}(\mathbf{v}, \mathbf{w})=\sqcup_{\mathbf{v}^{\prime}} \mathfrak{M}_{0}^{r e g}\left(\mathbf{v}^{\prime}, \mathbf{w}\right)
$$

whose restriction to each $\mathfrak{M}(\mathbf{v}, \mathbf{w})$ is $\pi_{\mathbf{v}}$.

Then, $\pi$ is a topological fiber bundle over each stratum $\mathfrak{M}_{0}^{\text {reg }}\left(\mathbf{v}^{\prime}, \mathbf{w}\right)$ [10]. Furthermore, according to [10, Corollary 10.11] the projective morphism $\pi$ is a strictly semi-small morphism with respect to the stratification given by (2.3).

\section{Nakajima's construction}

In the remaining part of this paper, we fix a dimension vector $\mathbf{w}=$ $\left(\operatorname{dim} W_{i}\right)_{i \in I}$. We also assume that all quiver varieties $\mathfrak{M}(\mathbf{v}, \mathbf{w})$ are obtained from the Dynkin diagrams $(I, E)$ of finite dimensional complex simple Lie algebras of type $A D E$. Also, $H_{i}(X)$ will always denote the Borel-Moore homology with $\mathbb{C}$-coefficients and $H_{i}^{\text {ord }}(X)$ stands for the ordinary homology. In particular, $H_{t o p}(X)$ indicates the Borel-Moore homology at the degree $\operatorname{dim}_{\mathbb{R}} X$. We refer to $[2,7]$ for the details of the Borel-Moore homology.

Let $\mathfrak{g}$ be a finite dimensional complex simple Lie algebra of type $A D E$, and let $\operatorname{rank}(\mathfrak{g})=n$. Then, the following data are attached to $\mathfrak{g}$ with respect to a Cartan subalgebra $\mathfrak{h}$ of $\mathfrak{g}$.

1. simple roots $\alpha_{k} \in \mathfrak{h}^{*}(k=1,2, \cdots, n)$.

2. simple coroots $h_{k} \in \mathfrak{h}(k=1,2, \cdots, n)$.

3. fundamental weights $\Lambda_{k} \in \mathfrak{h}^{*}(k=1,2, \cdots, n)$ such that $\Lambda_{k}\left(h_{l}\right)=$ $\delta_{k l}$.

4. Cartan matrix $C=\left(\alpha_{j}\left(h_{i}\right)\right)$.

We set $\alpha_{\mathbf{v}}=\sum_{k=1}^{n}\left(\operatorname{dim} V_{k}\right) \alpha_{k}$ and $\Lambda_{\mathbf{w}}=\sum_{k=1}^{n}\left(\operatorname{dim} W_{k}\right) \Lambda_{k}$ for given dimension vectors $\mathbf{v}=\left(\operatorname{dim} V_{1}, \cdots, \operatorname{dim} V_{n}\right)$ and $\mathbf{w}=\left(\operatorname{dim} W_{1}, \cdots\right.$, $\left.\operatorname{dim} W_{n}\right)$. We also write $\mathbf{e}^{k}$ for the dimension vector such that $k$ th entry is 1 and the other entries are 0 .

Lemma 3.1. Let $\mathfrak{g}$ be a finite dimensional complex simple Lie algebra of type $A D E$, and let $\mathfrak{M}(\mathbf{v}, \mathbf{w})$ be a quiver variety obtained from the Dynkin diagram of $\mathfrak{g}$. Then, $\mathfrak{M}(\mathbf{v}, \mathbf{w})$ is empty for all but finitely many choices of $\mathbf{v}$. 
Proof. We first notice that $\mathfrak{M}(\mathbf{v}, \mathbf{w}) \neq \emptyset$ if and only if $\mathfrak{L}(\mathbf{v}, \mathbf{w}) \neq \emptyset$.

Recall that $\bigoplus_{\mathbf{v}^{\prime}} H_{\text {top }}\left(\mathfrak{L}\left(\mathbf{v}^{\prime}, \mathbf{w}\right)\right)$ is the irreducible highest weight $\mathfrak{U}(\mathfrak{g})$-module, and each direct summand $H_{\text {top }}\left(\mathfrak{L}\left(\mathbf{v}^{\prime}, \mathbf{w}\right)\right)$ has a weight $\Lambda_{\mathbf{w}}-\alpha_{\mathbf{v}^{\prime}}[9,10]$.

So, $\bigoplus_{\mathbf{v}^{\prime}} H_{\text {top }}\left(\mathfrak{L}\left(\mathbf{v}^{\prime}, \mathbf{w}\right)\right)$ has the weight space $H_{\text {top }}(\mathfrak{L}(\mathbf{v}, \mathbf{w}))$ of weight $\Lambda_{\mathbf{w}}-\alpha_{\mathbf{v}}$ if $\mathfrak{L}(\mathbf{v}, \mathbf{w}) \neq \emptyset$. According to the basic representation theory of finite dimensional simple Lie algebras, every integrable highest weight $\mathfrak{U}(\mathfrak{g})$-module is finite dimensional. Thus the direct sum $\bigoplus_{\mathbf{v}^{\prime}} H_{t o p}\left(\mathfrak{L}\left(\mathbf{v}^{\prime}, \mathbf{w}\right)\right)$ is a finite direct sum. The lemma now follows.

For two dimension vectors $\mathbf{v}^{1}$ and $\mathbf{v}^{2}$ satisfying $\mathbf{v}^{1}=\mathbf{v}^{2}-\mathbf{e}^{k}$, we consider two quiver varieties $\mathfrak{M}\left(\mathbf{v}^{1}, \mathbf{w}\right)$ and $\mathfrak{M}\left(\mathbf{v}^{2}, \mathbf{w}\right)$. From these two quiver varieties, we define the Hecke correspondence $\mathfrak{B}_{k}\left(\mathbf{v}^{2}, \mathbf{w}\right)$ as follows: a point $\left(\left[B^{1}, i^{1}, j^{1}\right],\left[B^{2}, i^{2}, j^{2}\right]\right)$ of $\mathfrak{M}\left(\mathbf{v}^{1}, \mathbf{w}\right) \times \mathfrak{M}\left(\mathbf{v}^{2}, \mathbf{w}\right)$ is an element of $\mathfrak{B}_{k}\left(\mathbf{v}^{2}, \mathbf{w}\right)$ if and only if there exists $\zeta \in \bigoplus_{k \in I} \operatorname{Hom}\left(V_{k}^{1}, V_{k}^{2}\right)$ such that

$$
\zeta B^{1}=B^{2} \zeta, \zeta i^{1}=i^{2}, j^{1}=j^{2} \zeta .
$$

The stability condition implies that such $\zeta$ exists uniquely if it exists [10].

In virtue of the morphisms (2.2), we have the following Steinberg-type variety for given two dimension vectors $\mathbf{v}^{1}=\mathbf{v}^{2}-\mathbf{e}^{k}$ and $\mathbf{v}^{2}$ :

$$
Z\left(\mathbf{v}^{1}, \mathbf{v}^{2} ; \mathbf{w}\right)=\left\{\left(x^{1}, x^{2}\right) \in \mathfrak{M}\left(\mathbf{v}^{1}, \mathbf{w}\right) \times \mathfrak{M}\left(\mathbf{v}^{2}, \mathbf{w}\right) \mid \pi_{\mathbf{v}^{1}}\left(x^{1}\right)=\pi_{\mathbf{v}^{2}}\left(x^{2}\right)\right\} .
$$

From Equations (3.1), it is easy to see that $\mathfrak{B}_{k}\left(\mathbf{v}^{2}, \mathbf{w}\right)$ is a subvariety of $Z\left(\mathbf{v}^{1}, \mathbf{v}^{2} ; \mathbf{w}\right)$. In fact, $\mathfrak{B}_{k}(\mathbf{v}, \mathbf{w})$ is an irreducible component of $Z\left(\mathbf{v}^{1}, \mathbf{v}^{2} ; \mathbf{w}\right)$.

Let $\omega: \mathfrak{M}\left(\mathbf{v}^{1}, \mathbf{w}\right) \times \mathfrak{M}\left(\mathbf{v}^{2}, \mathbf{w}\right) \longrightarrow \mathfrak{M}\left(\mathbf{v}^{2}, \mathbf{w}\right) \times \mathfrak{M}\left(\mathbf{v}^{1}, \mathbf{w}\right)$ be the morphism which interchange the two factors, and let

$$
r_{k}(\mathbf{v}, \mathbf{w})=\frac{1}{2}\left(\operatorname{dim}_{\mathbb{C}} \mathfrak{M}\left(\mathbf{v}^{1}, \mathbf{w}\right)-\operatorname{dim}_{\mathbb{C}} \mathfrak{M}\left(\mathbf{v}^{2}, \mathbf{w}\right)\right) .
$$

Then, we define

$$
\begin{aligned}
E_{k} & =\sum_{\mathbf{v}}\left[\mathfrak{B}_{k}(\mathbf{v}, \mathbf{w})\right] \\
F_{k} & =\sum_{\mathbf{v}}(-1)^{r_{k}(\mathbf{v}, \mathbf{w})}\left[\omega\left(\mathfrak{B}_{k}(\mathbf{v}, \mathbf{w})\right)\right], \\
H_{k} & =\sum_{\mathbf{v}}\left(\Lambda_{\mathbf{w}}-\alpha_{\mathbf{v}}\right)\left(h_{k}\right)[\triangle(\mathbf{v}, \mathbf{w})],
\end{aligned}
$$


where $\triangle(\mathbf{v}, \mathbf{w})$ denotes the diagonal in $\mathfrak{M}(\mathbf{v}, \mathbf{w}) \times \mathfrak{M}(\mathbf{v}, \mathbf{w})$.

According to Lemma 3.1, the elements $E_{k}, F_{k}$ and $H_{k}$ are finite sums so that they are well-defined elements in $\bigoplus_{\mathbf{v}^{1}, \mathbf{v}^{2}} H_{\text {top }}\left(Z\left(\mathbf{v}^{1}, \mathbf{v}^{2} ; \mathbf{w}\right)\right)$.

We recall that $\bigoplus_{\mathbf{v}^{1}, \mathbf{v}^{2}} H_{\text {top }}\left(Z\left(\mathbf{v}^{1}, \mathbf{v}^{2} ; \mathbf{w}\right)\right)$ is a $\mathbb{C}$-algebra equipped with the convolution product, and this algebra yields the following convolution action

$$
\star: H_{\text {top }}\left(Z\left(\mathbf{v}^{1}, \mathbf{v}^{2}, \mathbf{w}\right)\right) \otimes H_{\text {top }}\left(\mathfrak{M}\left(\mathbf{v}^{2}, \mathbf{w}\right)_{x}\right) \longrightarrow H_{\text {top }}\left(\mathfrak{M}\left(\mathbf{v}^{1}, \mathbf{w}\right)_{x}\right)
$$

which is due to the strictly semi-small morphism $\pi$ in (2.4). We refer to $[10$, Section 8] for the details of the action (3.2).

Theorem 3.2. $\quad$ 1. There exists a unique algebra homomorphism $\Phi: \mathfrak{U}(\mathfrak{g}) \longrightarrow \bigoplus_{\mathbf{v}^{1}, \mathbf{v}^{2}} H_{\text {top }}\left(Z\left(\mathbf{v}^{1}, \mathbf{v}^{2} ; \mathbf{w}\right)\right)$ such that $\Phi\left(e_{k}\right)=E_{k}$, $\Phi\left(f_{k}\right)=F_{k}$ and $\Phi\left(h_{k}\right)=H_{k}$, where $\left\{e_{k}, f_{k}, h_{k}\right\}_{k=1, \cdots, n}$ is the set of Chevalley generators of $\mathfrak{g}$.

2. Let $x \in \mathfrak{M}_{0}^{\text {reg }}\left(\mathbf{v}^{0}, \mathbf{w}\right)$. Then, $\bigoplus_{\mathbf{v}} H_{\text {top }}\left(\mathfrak{M}(\mathbf{v}, \mathbf{w})_{x}\right)$ is the irreducible highest weight $\mathfrak{U}(\mathfrak{g})$-module with the highest weight vector $\left[\mathfrak{M}\left(\mathbf{v}^{0}, \mathbf{w}\right)_{x}\right]$.

3. The algebra homomorphism $\Phi$ in (1) is surjective.

Proof. See [10, Theorem 9.4, Theorem 10.2, Theorem 10.15] for the proofs.

Since the homomorphism $\Phi$ is surjective, we obtain the following corollary.

Corollary 3.3. Any simple $\bigoplus_{\mathbf{v}^{1}, \mathbf{v}^{2}} H_{t o p}\left(Z\left(\mathbf{v}^{1}, \mathbf{v}^{2} ; \mathbf{w}\right)\right)$-module yields an irreducible representation of $\mathfrak{U}(\mathfrak{g})$.

\section{4. sheaf-theoretic approach}

In this section, we apply the Beilinson-Bernstein-Deligne decomposition theorem to the morphism $\pi$ in (2.4) (see [1] for the details of the decomposition theorem). In particular, in our case where $\pi$ is strictly semi-small, we have the following nice form of the Beilinson-BernsteinDeligne decomposition theorem:

$$
\pi_{*} \mathcal{C}=\bigoplus_{\varphi=\left(\mathfrak{M}_{0}^{r e g}\left(\mathbf{v}^{\varphi}, \mathbf{w}\right), \chi_{\varphi}\right)} L_{\varphi} \otimes I C_{\varphi},
$$

where $\mathcal{C}$ is the constant perverse sheaf on $\sqcup_{\mathbf{v}} \mathfrak{M}(\mathbf{v}, \mathbf{w})$ such that $\left.\mathcal{C}\right|_{\mathfrak{M}(\mathbf{v}, \mathbf{w})}$ $=\underline{\mathbb{C}}_{\mathfrak{M}(\mathbf{v}, \mathbf{w})}\left[\operatorname{dim}_{\mathbb{C}} \mathfrak{M}(\mathbf{v}, \mathbf{w})\right]$, and the direct sum is taken over the pairs 
consisting of a stratum $\mathfrak{M}_{0}^{r e g}\left(\mathbf{v}^{\varphi}, \mathbf{w}\right)$ of $\mathfrak{M}_{0}(\mathbf{v}, \mathbf{w})$ and an irreducible local system $\chi_{\varphi}$ on $\mathfrak{M}_{0}^{r e g}\left(\mathbf{v}^{\varphi}, \mathbf{w}\right)$.

According to [9, Corollary 5.5], we have a homotopy equivalence $i^{\mathbf{v}}$ : $\mathfrak{L}(\mathbf{v}, \mathbf{w}) \hookrightarrow \mathfrak{M}(\mathbf{v}, \mathbf{w})$. This homotopy equivalence is proper because $\mathfrak{L}(\mathbf{v}, \mathbf{w})$ is compact. Thus, $i^{\mathbf{v}}$ yields the direct image map between induced Borel-Moore homology spaces.

Let $i_{*}: \bigoplus_{\mathbf{v}} H_{2 d_{\mathbf{v}}}(\mathfrak{L}(\mathbf{v}, \mathbf{w})) \longrightarrow \bigoplus_{\mathbf{v}} H_{2 d_{\mathbf{v}}}(\mathfrak{M}(\mathbf{v}, \mathbf{w}))$ be a direct sum of the direct image maps between the Borel-Moore homologies. That is, we have $i_{*}=\bigoplus_{\mathbf{v}} i_{*}^{\mathbf{v}}$ for $i_{*}^{\mathbf{v}}: H_{2 d_{\mathbf{v}}}(\mathfrak{L}(\mathbf{v}, \mathbf{w})) \longrightarrow H_{2 d_{\mathbf{v}}}(\mathfrak{M}(\mathbf{v}, \mathbf{w}))$.

Theorem 4.1. Let us keep the same notations as above. Then, we have

$$
\operatorname{Im}\left(i_{*}\right)=\bigoplus_{\mathbf{v}} H_{2 d_{\mathbf{v}}}(\mathfrak{L}(\mathbf{v}, \mathbf{w}))=L_{\left(\{0\}, \mathbb{C}_{\{0\}}\right)} .
$$

Proof. We notice that $\mathfrak{M}_{0}^{\text {reg }}(0, \mathbf{w})=\{0\}$. That is, $\mathfrak{M}_{0}^{\text {reg }}(0, \mathbf{w})$ is an one point stratum. Let $i_{\{0\}}:\{0\} \hookrightarrow \mathfrak{M}_{0}(\mathbf{v}, \mathbf{w})$ be the embedding of the one point stratum $\mathfrak{M}_{0}^{r e g}(0, \mathbf{w})$. Then, by [3, Lemma 8.5.4] and the Beilinson-Bernstein-Deligne decomposition theorem we have

$$
\begin{aligned}
H_{k}(\mathfrak{L}(\mathbf{v}, \mathbf{w})) & =H^{2 d_{\mathbf{v}}-k}\left(i_{\{0\}}^{!} \pi_{\mathbf{v} *} \mathbb{C}_{\mathfrak{M}(\mathbf{v}, \mathbf{w})}\left[2 d_{\mathbf{v}}\right]\right) \\
& =\bigoplus_{\varphi} L_{\varphi} \otimes H^{2 d_{\mathbf{v}}-k}\left(i_{\{0\}}^{!} I C_{\varphi}\right)
\end{aligned}
$$

where $\varphi=\left(\mathfrak{M}_{0}^{\text {reg }}\left(\mathbf{v}^{\varphi}, \mathbf{w}\right), \chi_{\varphi}\right)$ is a pair associated with both $\mathbf{v}^{\varphi} \leq \mathbf{v}$ and an irreducible local system $\chi_{\varphi}$ on $\mathfrak{M}_{0}^{\text {reg }}\left(\mathbf{v}^{\varphi}, \mathbf{w}\right)$.

Similarly, due to [3, Lemma 8.5.4] and the Beilinson-Bernstein-Deligne decomposition theorem, we obtain

$$
\begin{aligned}
H_{k}(\mathfrak{M}(\mathbf{v}, \mathbf{w})) & =H^{4 d_{\mathbf{v}}-k}(\mathfrak{M}(\mathbf{v}, \mathbf{w})) \\
& =H^{4 d_{\mathbf{v}}-k}(\mathfrak{L}(\mathbf{v}, \mathbf{w})) \\
& =\bigoplus_{\varphi} L_{\varphi} \otimes H^{2 d_{\mathbf{v}}-k}\left(i_{\{0\}}^{*} I C_{\varphi}\right)
\end{aligned}
$$

where $\varphi$ is the same as that of (4.3). Thus, we can identify

$$
i_{*}^{\mathbf{v}}: H_{2 d_{\mathbf{v}}}(\mathfrak{L}(\mathbf{v}, \mathbf{w})) \longrightarrow H_{2 d_{\mathbf{v}}}(\mathfrak{M}(\mathbf{v}, \mathbf{w}))
$$

with a direct sum of morphisms

$$
\Phi_{\varphi}: L_{\varphi} \otimes H^{0}\left(i_{\{0\}}^{!} I C_{\varphi}\right) \longrightarrow L_{\varphi} \otimes H^{0}\left(i_{\{0\}}^{*} I C_{\varphi}\right),
$$

where $\varphi=\left(\mathfrak{M}_{0}^{r e g}\left(\mathbf{v}^{\varphi}, \mathbf{w}\right), \chi_{\varphi}\right)$ for $\mathbf{v}^{\varphi} \leq \mathbf{v}$. 
Recall from [3, Lemma 8.5.3] that $H^{0}\left(i_{\{0\}}^{!} I C_{\varphi}\right)=0$ for $\varphi=\left(\mathfrak{M}_{0}^{\text {reg }}\right.$ $\left.\left(\mathbf{v}^{\varphi}, \mathbf{w}\right), \chi_{\varphi}\right)$ satisfying $\mathfrak{M}_{0}^{\text {reg }}\left(\mathbf{v}^{\varphi}, \mathbf{w}\right) \neq \mathfrak{M}_{0}^{r e g}(0, \mathbf{w})$. Moreover, if $\mathfrak{M}_{0}^{\text {reg }}$ $\left(\mathbf{v}^{\varphi}, \mathbf{w}\right)=\mathfrak{M}_{0}^{r e g}(0, \mathbf{w})$ then the corresponding morphisms $\Phi_{\varphi}$ is an isomorphism.

Hence, we obtain that $\operatorname{ker}\left(i_{*}^{\mathbf{v}}\right)=\{0\}$. Furthermore, we notice that $H^{0}\left(i_{\{0\}}^{*} I C_{\varphi}\right)=\mathbb{C}$ for $\varphi=\left(\mathfrak{M}_{0}^{\text {reg }}(0, \mathbf{w}), \mathbb{C}_{\{0\}}\right)$. The theorem is now immediate.

Henceforth, we write $\mathfrak{M}(\mathbf{w})=\sqcup_{\mathbf{v}} \mathfrak{M}(\mathbf{v}, \mathbf{w}), \mathfrak{L}(\mathbf{w})=\sqcup_{\mathbf{v}} \mathfrak{L}(\mathbf{v}, \mathbf{w})$ and $Z(\mathbf{w})=\sqcup_{\mathbf{v}^{1}, \mathbf{v}^{2}} Z\left(\mathbf{v}^{1}, \mathbf{v}^{2} ; \mathbf{w}\right)$.

We now introduce a new $\mathbb{Z}$-grading on the Borel-Moore homology to preserve gradings under the convolution product. More explicitly, we set

$$
\begin{gathered}
H_{[p]}(\mathfrak{L}(\mathbf{v}, \mathbf{w})):=H_{2 d_{\mathbf{v}}-p}(\mathfrak{L}(\mathbf{v}, \mathbf{w})), \\
H_{[p]}(\mathfrak{L}(\mathbf{w})):=\bigoplus_{\mathbf{v}} H_{[p]}(\mathfrak{L}(\mathbf{v}, \mathbf{w})), \\
H_{[p]}\left(Z\left(\mathbf{v}^{1}, \mathbf{v}^{2} ; \mathbf{w}\right)\right):=H_{m_{\mathbf{v}^{1}}+m_{\mathbf{v}^{2}}-p}\left(Z\left(\mathbf{v}^{1}, \mathbf{v}^{2} ; \mathbf{w}\right)\right)
\end{gathered}
$$

and

$$
H_{[p]}(Z(\mathbf{w})):=\bigoplus_{\mathbf{v}^{1}, \mathbf{v}^{2}} H_{[p]}\left(Z\left(\mathbf{v}^{1}, \mathbf{v}^{2} ; \mathbf{w}\right)\right)
$$

where $d_{\mathbf{v}}=\operatorname{dim}_{\mathbb{C}} \mathfrak{L}(\mathbf{v}, \mathbf{w})$ and $m_{\mathbf{v}^{i}}=\operatorname{dim}_{\mathbb{C}} \mathfrak{M}\left(\mathbf{v}^{i}, \mathbf{w}\right)$.

Then, by the definition of the convolution product we have

$$
H_{[p]}\left(Z\left(\mathbf{v}^{1}, \mathbf{v}^{2} ; \mathbf{w}\right)\right) \star H_{[q]}\left(Z\left(\mathbf{v}^{2}, \mathbf{v}^{3} ; \mathbf{w}\right)\right) \subset H_{[p+q]}\left(Z\left(\mathbf{v}^{1}, \mathbf{v}^{3} ; \mathbf{w}\right)\right),
$$

and this implies that

$$
H_{[p]}(Z(\mathbf{w})) \star H_{[q]}(Z(\mathbf{w})) \subset H_{[p+q]}(Z(\mathbf{w})) .
$$

Let $D^{b}(\operatorname{sh}(X))$ be the bounded derived category of sheaves on an algebraic variety $X$. Then, we write $D^{b}(X)$ for the full subcategory of $D^{b}(\operatorname{sh}(X))$ formed by constructible complexes. For any complexes $A^{\bullet}$, $B^{\bullet} \in D^{b}(X), \operatorname{Ext}_{D^{b}(X)}^{k}\left(A^{\bullet}, B^{\bullet}\right)$ is defined as $\operatorname{Hom}_{D^{b}(X)}\left(A^{\bullet}, B^{\bullet}[k]\right)$.

With the new gradings defined in above, we obtain the following theorem.

Theorem 4.2. $\quad$ 1. The convolution action makes $\bigoplus_{p \geq 0} H_{[p]}(\mathfrak{L}(\mathbf{w}))$ a graded $\bigoplus_{p \geq 0} H_{[p]}(Z(\mathbf{w}))$-module with respect to the new gradings defined in above. 
2. We have a graded algebra isomorphism

$$
\bigoplus_{p \geq 0}\left(H_{[p]}(Z(\mathbf{w}))\right) \simeq \bigoplus_{p \geq 0}\left(\bigoplus_{\varphi, \psi} \operatorname{Hom}_{\mathbb{C}}\left(L_{\varphi}, L_{\psi}\right) \otimes \operatorname{Ext}_{D^{b}\left(\cup_{\mathbf{v}} \mathfrak{M}_{0}(\mathbf{v}, \mathbf{w})\right)}\left(I C_{\varphi}, I C_{\psi}\right)\right),
$$

where $\varphi$ and $\psi$ are the pairs arising from the decomposition (4.1). In particular, we obtain that

$$
\bigoplus_{\mathbf{v}^{1}, \mathbf{v}^{2}} H_{t o p}\left(Z\left(\mathbf{v}^{1}, \mathbf{v}^{2} ; \mathbf{w}\right)\right) \simeq \bigoplus_{\varphi} \operatorname{End}\left(L_{\varphi}\right) .
$$

Proof. The first part of the theorem is obtained directly from the definition of the convolution product. In more details, we consider the convolution product under the circumstances $M_{1}=\mathfrak{M}\left(\mathbf{v}^{1}, \mathbf{w}\right), M_{2}=$ $\mathfrak{M}\left(\mathbf{v}^{2}, \mathbf{w}\right), M_{3}=\{$ point $\}, Z\left(\mathbf{v}^{1}, \mathbf{v}^{2} ; \mathbf{w}\right) \subset M_{1} \times M_{2}$ and $\mathfrak{L}\left(\mathbf{v}^{2}, \mathbf{w}\right) \subset$ $M_{2} \times M_{3}$. Then, by the definition of the convolution product, we obtain that

$H_{m_{\mathbf{v}^{1}}+m_{\mathbf{v}^{2}}-p}\left(Z\left(\mathbf{v}^{1}, \mathbf{v}^{2} ; \mathbf{w}\right)\right) \star H_{m_{\mathbf{v}^{2}}-q}\left(\mathfrak{L}\left(\mathbf{v}^{2}, \mathbf{w}\right)\right) \subset H_{m_{\mathbf{v}^{1}}-(p+q)}\left(\mathfrak{L}\left(\mathbf{v}^{1}, \mathbf{w}\right)\right)$.

This implies that

$$
H_{[p]}\left(Z\left(\mathbf{v}^{1}, \mathbf{v}^{2} ; \mathbf{w}\right)\right) \star H_{[q]}\left(\mathfrak{L}\left(\mathbf{v}^{2}, \mathbf{w}\right)\right) \subset H_{[p+q]}\left(\mathfrak{L}\left(\mathbf{v}^{1}, \mathbf{w}\right)\right) .
$$

We now prove the second part of the theorem.

Let $\pi: \mathfrak{M}(\mathbf{w}) \longrightarrow \cup_{\mathbf{v}} \mathfrak{M}_{0}(\mathbf{v}, \mathbf{w})$ be the projective morphism defined in (2.4). Then, by [3, Lemma 8.6.1] and the decomposition (4.1), we obtain that

$$
\begin{aligned}
H_{[p]}(Z(\mathbf{w})) & \simeq \operatorname{Ext}_{D^{b}\left(\cup_{\mathbf{v}} \mathfrak{M}_{0}(\mathbf{v}, \mathbf{w})\right)}^{p}\left(\pi_{*} \mathcal{C}, \pi_{*} \mathcal{C}\right) \\
& \simeq \operatorname{Ext}_{D^{b}\left(\cup_{\mathbf{v}} \mathfrak{M}_{0}(\mathbf{v}, \mathbf{w})\right)}^{p}\left(\bigoplus_{\varphi} L_{\varphi} \otimes I C_{\varphi}, \bigoplus_{\psi} L_{\psi} \otimes I C_{\psi}\right) \\
& \simeq \bigoplus_{\varphi, \psi} \operatorname{Hom}_{\mathbb{C}}\left(L_{\varphi}, L_{\psi}\right) \otimes \operatorname{Ext}_{D^{b}\left(\cup_{\mathbf{v}} \mathfrak{M}_{0}(\mathbf{v}, \mathbf{w})\right)}^{p}\left(I C_{\varphi}, I C_{\psi}\right)
\end{aligned}
$$

where $\varphi=\left(\mathfrak{M}_{0}^{r e g}\left(\mathbf{v}^{\varphi}, \mathbf{w}\right), \chi_{\varphi}\right)\left(\right.$ resp., $\left.\psi=\left(\mathfrak{M}_{0}^{r e g}\left(\mathbf{v}^{\psi}, \mathbf{w}\right), \chi_{\psi}\right)\right)$ is a pair associated with an irreducible local system $\chi_{\varphi}$ (resp., $\left.\chi_{\psi}\right)$ on $\mathfrak{M}_{0}^{r e g}\left(\mathbf{v}^{\varphi}, \mathbf{w}\right)$ (resp., $\left.\mathfrak{M}_{0}^{r e g}\left(\mathbf{v}^{\psi}, \mathbf{w}\right)\right)$.

Since the Yoneda product is compatible with the convolution, the above isomorphisms yield a graded algebra isomorphism. In addition, we also have

$$
\begin{aligned}
\bigoplus_{\mathbf{v}^{1}, \mathbf{v}^{2}} H_{t o p}\left(Z\left(\mathbf{v}^{1}, \mathbf{v}^{2} ; \mathbf{w}\right)\right) & =\bigoplus_{\mathbf{v}^{1}, \mathbf{v}^{2}} H_{m_{\mathbf{v}^{1}}+m_{\mathbf{v}^{2}}}\left(Z\left(\mathbf{v}^{1}, \mathbf{v}^{2} ; \mathbf{w}\right)\right) \\
& =H_{[0]}(Z(\mathbf{w}))
\end{aligned}
$$


The theorem is now immediate because $\operatorname{Ext}_{D^{b}\left(\cup_{\mathbf{v}} \mathfrak{M}_{0}(\mathbf{v}, \mathbf{w})\right)}^{0}\left(I C_{\varphi}, I C_{\psi}\right)=$ $\mathbb{C} \delta_{\varphi, \psi}$.

By Equation (4.4), each $L_{\varphi}$ is equipped with $\bigoplus_{\mathbf{v}^{1}, \mathbf{v}^{2}} H_{t o p}\left(Z\left(\mathbf{v}^{1}, \mathbf{v}^{2}\right.\right.$; w))-module structure.

Corollary 4.3. The vector spaces $L_{\varphi}$ in (4.4) are simple $\mathfrak{U}(\mathfrak{g})$-modules.

Proof. Notice that all simple modules of the semisimple algebra $\bigoplus_{\varphi} \operatorname{End}\left(L_{\varphi}\right)$ are of the form $L_{\varphi}$. Thus, the collection $\left\{L_{\varphi}\right\}$ is a complete list of simple $\bigoplus_{\mathbf{v}^{1}, \mathbf{v}^{2}} H_{\text {top }}\left(Z\left(\mathbf{v}^{1}, \mathbf{v}^{2} ; \mathbf{w}\right)\right)$-modules. The corollary now follows from Corollary 3.3.

Remark 4.4. 1. Equation (4.4) may be viewed as a sheaf-theoretic analogue of [10, Theorem 10.15].

2. Let $\Phi$ be the surjective algebra homomorphism in Theorem 3.2, and let us denote by $J(R)$ the Jacobson radical of a ring $R$. Then, we obtain that $J(\mathfrak{U}(\mathfrak{g})) \subset K \operatorname{Ker}(\Phi)$ because

$$
\Phi(J(\mathfrak{U}(\mathfrak{g}))) \subset J\left(\bigoplus_{\mathbf{v}^{1}, \mathbf{v}^{2}} H_{\text {top }}\left(Z\left(\mathbf{v}^{1}, \mathbf{v}^{2} ; \mathbf{w}\right)\right)\right)=\{0\} .
$$

For this reason, in general we may have a simple $\mathfrak{U}(\mathfrak{g})$-module that does not appear on the complete list $\left\{L_{\varphi}\right\}$ of simple $\bigoplus_{\mathbf{v}^{1}, \mathbf{v}^{2}} H_{t o p}(Z$ $\left.\left(\mathbf{v}^{1}, \mathbf{v}^{2} ; \mathbf{w}\right)\right)$-modules.

In the following theorem, we provide a refined form of the decomposition (4.1).

Theorem 4.5. Let $\pi$ be the projective morphism defined in (2.4). Then, the intersection cohomology complexes that appear in the decomposition (4.1) are exactly those associated with the constant local system $\mathbb{C}_{\mathfrak{M}_{0}^{\text {reg }}\left(\mathbf{v}^{0}, \mathbf{w}\right)}$ on each stratum $\mathfrak{M}_{0}^{\text {reg }}\left(\mathbf{v}^{0}, \mathbf{w}\right)$.

In other words, the decomposition (4.1) can be refined as

$$
\pi_{*} \mathcal{C}=\underset{\varphi=\left(\mathfrak{M}_{0}^{r e g}\left(\mathbf{v}^{\varphi}, \mathbf{w}\right), \mathbb{C}_{\mathfrak{M}_{0}^{r e g}\left(\mathbf{v}^{\varphi}, \mathbf{w}\right)}\right)}{\bigoplus} L_{\varphi} \otimes I C_{\varphi}
$$

Proof. We first notice that any nonempty stratum $\mathfrak{M}_{0}^{\text {reg }}(\mathbf{v}, \mathbf{w})$ is irreducible because $\mathfrak{M}_{0}^{\text {reg }}(\mathbf{v}, \mathbf{w})$ is an open subset of $\mathfrak{M}_{0}(\mathbf{v}, \mathbf{w})$ [4]. So, $\mathbb{C}_{\mathfrak{M}_{0}^{r e g}(\mathbf{v} \varphi, \mathbf{w})}$ is an irreducible local system on each nonempty stratum $\mathfrak{M}_{0}^{r e g}\left(\mathbf{v}^{\varphi}, \mathbf{w}\right)$.

Let us now take $x \in \mathfrak{M}_{0}^{r e g}\left(\mathbf{v}^{0}, \mathbf{w}\right)$. 
By applying the same argument as the proof of Theorem 4.1 to the embedding $j: \mathfrak{M}_{0}^{r e g}(\mathbf{v}, \mathbf{w}) \hookrightarrow \mathfrak{M}_{0}(\mathbf{v}, \mathbf{w})$, we have

$$
\bigoplus_{\mathbf{v}} H_{\text {top }}\left(\mathfrak{M}(\mathbf{v}, \mathbf{w})_{x}\right)=\bigoplus_{\varphi} L_{\varphi}
$$

where $L_{\varphi}$ are vector spaces that appear in the decomposition (4.1) and $\varphi$ runs over such $\left(\mathfrak{M}_{0}^{\text {reg }}\left(\mathbf{v}^{0}, \mathbf{w}\right), \chi\right)$ that consist of the fixed stratum $\mathfrak{M}_{0}^{r e g}\left(\mathbf{v}^{0}, \mathbf{w}\right)$ containing $x$ and irreducible local systems $\chi$ attached to $\mathfrak{M}_{0}^{\text {reg }}\left(\mathbf{v}^{0}, \mathbf{w}\right)$.

However, by Theorem 3.2, the left hand side of (4.5) is an irreducible $\mathfrak{U}(\mathfrak{g})$-module. This implies that we should have only one direct summand on the right hand side of (4.5) because $\left\{L_{\varphi}\right\}$ is a set of simple $\mathfrak{U}(\mathfrak{g})$-modules due to Corollary 4.3. Hence, we obtain

$$
\left.\bigoplus_{\mathbf{v}} H_{\text {top }}\left(\mathfrak{M}(\mathbf{v}, \mathbf{w})_{x}\right)=L_{\left(\mathfrak{M}_{0}^{r e g}\left(\mathbf{v}^{0}, \mathbf{w}\right), \underline{\mathbb{C}}_{\mathfrak{M}_{0}^{r e g}}\left(\mathbf{v}^{0}, \mathbf{w}\right)\right.}\right) \cdot
$$

Therefore, the intersection cohomology complexes that appear in the decomposition of $\pi_{*} \mathcal{C}$ are those coming from the pairs $\left(\mathfrak{M}_{0}^{\text {reg }}\left(\mathbf{v}^{0}, \mathbf{w}\right)\right.$, $\left.\mathbb{C}_{\mathfrak{M}_{0}^{r e g}\left(\mathbf{v}^{0}, \mathbf{w}\right)}\right)$ for each nonempty stratum $\mathfrak{M}_{0}^{\text {reg }}\left(\mathbf{v}^{0}, \mathbf{w}\right)$. The theorem now follows.

\section{5. constructible functions}

Let $X$ be an algebraic variety. A constructible subset of $X$ is a finite union of locally closed subsets of $X$. A function $f: X \longrightarrow \mathbb{C}$ is called a constructible function if $f(X)$ is finite and $f^{-1}(a)$ is a constructible subset of $X$ for each $a \in \mathbb{C}$.

We write $A(X)$ for the $\mathbb{C}$-vector space consisting of all $\mathbb{C}$-valued constructible functions on $X$. If $Y$ is a subvariety of $X$, then we consider $A(Y)$ as a subspace of $A(X)$ by extending with zero on a set $X-Y$.

By the characteristic function $<Z>$ of a constructible subset $Z \subset X$ we mean

$$
<Z>(x)= \begin{cases}1, & \text { if } x \in Z, \\ 0, & \text { if } x \in X-Z .\end{cases}
$$

Then, the characteristic function $\langle Z\rangle$ is an element of $A(X)$. For a given morphism $p: X \longrightarrow Y$ between algebraic varieties, we define the push-forward $p_{*}: A(X) \longrightarrow A(Y)$ by

$$
\left(p_{*} f\right)(y)=\sum_{c \in \mathbb{C}} c \chi\left(p^{-1}(y) \cap f^{-1}(c)\right)
$$


where $\chi$ denotes the Euler characteristic. We also define the pull-back $p^{*}: A(Y) \longrightarrow A(X)$ by

$$
\left(p^{*} g\right)(x)=g(p(x)) \text {. }
$$

Let $M_{1}, M_{2}$ and $M_{3}$ be algebraic varieties, and let $p_{i j}: M_{1} \times M_{2} \times$ $M_{3} \longrightarrow M_{i} \times M_{j}(i, j=1,2,3)$ be the projections. Then, for $f \in$ $A\left(M_{1} \times M_{2}\right)$ and $g \in A\left(M_{2} \times M_{3}\right)$, we define the convolution product $f \star g$ by

$$
f \star g=\left(p_{13}\right)_{*}\left(\left(p_{12}^{*} f\right)\left(p_{23}^{*} g\right)\right) \in A\left(M_{1} \times M_{3}\right) .
$$

Suppose that we have morphisms $p_{i}: M_{i} \longrightarrow M_{0}(i=1,2,3)$ for a fixed variety $M_{0}$. By considering $A\left(M_{i} \times M_{0} M_{j}\right)$ as a subspaces of $A\left(M_{i} \times M_{j}\right)$, we define the convolution product $f \star g \in A\left(M_{1} \times M_{3}\right)$ for $f \in A\left(M_{1} \times M_{0} M_{2}\right)$ and $g \in A\left(M_{2} \times M_{0} M_{3}\right)$. Notice that the convolution $f \star g$ is also contained in $A\left(M_{1} \times_{M_{0}} M_{3}\right)$. In particular, we have the convolution product

$$
A\left(Z\left(\mathbf{v}^{1}, \mathbf{v}^{2} ; \mathbf{w}\right)\right) \otimes A\left(Z\left(\mathbf{v}^{2}, \mathbf{v}^{3} ; \mathbf{w}\right)\right) \longrightarrow A\left(Z\left(\mathbf{v}^{1}, \mathbf{v}^{3} ; \mathbf{w}\right)\right),
$$

and this convolution yields a $\mathbb{C}$-algebra structure on $\bigoplus_{\mathbf{v}^{1}, \mathbf{v}^{2}} A\left(Z\left(\mathbf{v}^{1}, \mathbf{v}^{2}\right.\right.$; $\mathbf{w})$ ).

Using Lemma 3.1, we define the following elements:

$$
\begin{aligned}
\widetilde{E_{k}} & =\sum_{\mathbf{v}}\left\langle\mathfrak{B}_{k}(\mathbf{v}, \mathbf{w})\right\rangle \in \underset{\mathbf{v}^{1}, \mathbf{v}^{2}}{\bigoplus} A\left(Z\left(\mathbf{v}^{1}, \mathbf{v}^{2} ; \mathbf{w}\right)\right), \\
\widetilde{F_{k}} & =\sum_{\mathbf{v}}\left\langle\omega\left(\mathfrak{B}_{k}(\mathbf{v}, \mathbf{w})\right)\right\rangle \in \underset{\mathbf{v}^{1}, \mathbf{v}^{2}}{\bigoplus} A\left(Z\left(\mathbf{v}^{1}, \mathbf{v}^{2} ; \mathbf{w}\right)\right), \\
\widetilde{H_{k}} & =\sum_{\mathbf{v}}\left(\Lambda_{\mathbf{w}}-\alpha_{\mathbf{v}}\right)\left(h_{k}\right)\langle\triangle(\mathbf{v}, \mathbf{w})\rangle \in \underset{\mathbf{v}^{1}, \mathbf{v}^{2}}{\bigoplus} A\left(Z\left(\mathbf{v}^{1}, \mathbf{v}^{2} ; \mathbf{w}\right)\right) .
\end{aligned}
$$

The following theorem is an analogue of Theorem 3.2 through constructible functions.

Theorem 5.1. There exists a unique algebra homomorphism

$$
\Psi: \mathfrak{U}(\mathfrak{g}) \longrightarrow \bigoplus_{\mathbf{v}^{1}, \mathbf{v}^{2}} A\left(Z\left(\mathbf{v}^{1}, \mathbf{v}^{2} ; \mathbf{w}\right)\right)
$$

such that $\Psi\left(e_{k}\right)=\widetilde{E_{k}}, \Psi\left(f_{k}\right)=\widetilde{F_{k}}$ and $\Psi\left(h_{k}\right)=\widetilde{H_{k}}$.

Proof. See [12, Theorem 6.5].

Put $M_{1}=\mathfrak{M}\left(\mathbf{v}^{1}, \mathbf{w}\right), M_{2}=\mathfrak{M}\left(\mathbf{v}^{2}, \mathbf{w}\right), M_{3}=\{$ point $\}, Z_{12}=$ $Z\left(\mathbf{v}^{1}, \mathbf{v}^{2} ; \mathbf{w}\right) \subset M_{1} \times M_{2}$ and $Z_{23}=\mathfrak{M}\left(\mathbf{v}^{2}, \mathbf{w}\right)_{x} \subset M_{2} \times M_{3}$ in the convolution set-up of constructible functions. 
Then, we obtain the convolution action

$$
A\left(Z\left(\mathbf{v}^{1}, \mathbf{v}^{2} ; \mathbf{w}\right)\right) \otimes A\left(\mathfrak{M}\left(\mathbf{v}^{2}, \mathbf{w}\right)_{x}\right) \longrightarrow A\left(\mathfrak{M}\left(\mathbf{v}^{1}, \mathbf{w}\right)_{x}\right) .
$$

So, the enveloping algebra $\mathfrak{U}(\mathfrak{g})$ acts on $\bigoplus_{\mathbf{v}} A\left(\mathfrak{M}(\mathbf{v}, \mathbf{w})_{x}\right)$ via the algebra homomorphism in Theorem 5.1.

In the following theorem, we give a constructible function analogue of [10, Theorem 10.2].

Theorem 5.2. Let $x \in \mathfrak{M}_{0}^{r e g}\left(\mathbf{v}^{0}, \mathbf{w}\right)$, and let

$$
\mathbb{L}(\mathbf{v}, \mathbf{w})_{x}=A\left(\mathfrak{M}(\mathbf{v}, \mathbf{w})_{x}\right) \cap\left(\mathfrak{U}(\mathfrak{g})\left\langle\mathfrak{M}\left(\mathbf{v}^{0}, \mathbf{w}\right)_{x}\right\rangle\right) .
$$

Then $\bigoplus_{\mathbf{v}} \mathbb{L}(\mathbf{v}, \mathbf{w})_{x}$ is the irreducible highest weight module with the highest weight vector $\left\langle\mathfrak{M}\left(\mathbf{v}^{0}, \mathbf{w}\right)_{x}\right\rangle$ of the highest weight $\Lambda_{\mathbf{w}}-\alpha_{\mathbf{v}^{0}}$.

Proof. By the convolution action defined in (5.2), we have

$$
{\widetilde{F_{k}}}^{N} \cdot A\left(\mathfrak{M}\left(\mathbf{v}^{0}, \mathbf{w}\right)_{x}\right) \subset A\left(\mathfrak{M}\left(\mathbf{v}^{0}+N \mathbf{e}^{k}, \mathbf{w}\right)_{x}\right)
$$

and

$$
{\widetilde{E_{k}}}^{N} \cdot A\left(\mathfrak{M}\left(\mathbf{v}^{0}, \mathbf{w}\right)_{x}\right) \subset A\left(\mathfrak{M}\left(\mathbf{v}^{0}-N \mathbf{e}^{k}, \mathbf{w}\right)_{x}\right) .
$$

From (5.3) and (5.4), we have $\bigoplus_{\mathbf{v}} \mathbb{L}(\mathbf{v}, \mathbf{w})_{x}=\mathfrak{U}(\mathfrak{g})\left\langle\mathfrak{M}\left(\mathbf{v}^{0}, \mathbf{w}\right)_{x}\right\rangle$.

Also, by Lemma 3.1, $\widetilde{F}_{k}^{N}$ and ${\widetilde{E_{k}}}^{N}$ are integrable operators on $\bigoplus_{\mathbf{v}} A$ $\left(\mathfrak{M}(\mathbf{v}, \mathbf{w})_{x}\right)$. This implies that $\bigoplus_{\mathbf{v}} \mathbb{L}(\mathbf{v}, \mathbf{w})_{x}$ is an integrable $\mathfrak{U}(\mathfrak{g})$ module.

On the other hand, we note that

$$
\widetilde{E_{k}} \cdot\left\langle\mathfrak{M}\left(\mathbf{v}^{0}, \mathbf{w}\right)_{x}\right\rangle \in A\left(\mathfrak{M}\left(\mathbf{v}^{0}-\mathbf{e}^{k}, \mathbf{w}\right)_{x}\right) .
$$

Since $x \in \mathfrak{M}_{0}^{\text {reg }}\left(\mathbf{v}^{0}, \mathbf{w}\right)$, we obtain $x \notin \mathfrak{M}_{0}\left(\mathbf{v}^{0}-\mathbf{e}^{k}, \mathbf{w}\right)$ due to the stratification (2.3). Thus, we have $\mathfrak{M}\left(\mathbf{v}^{0}-\mathbf{e}^{k}, \mathbf{w}\right)_{x}=\emptyset$ which implies that $\widetilde{E_{k}} \cdot\left\langle\mathfrak{M}\left(\mathbf{v}^{0}, \mathbf{w}\right)_{x}\right\rangle=0$.

Let us now take $\alpha \in \mathbb{L}(\mathbf{v}, \mathbf{w})_{x}$. Then, we have

$$
\widetilde{H_{k}} \cdot \alpha=\left(\Lambda_{\mathbf{w}}-\alpha_{\mathbf{v}}\right)\left(h_{k}\right) \alpha,
$$

because $\alpha \in A\left(\mathfrak{M}(\mathbf{v}, \mathbf{w})_{x}\right)$ and $\langle\triangle(\mathbf{v}, \mathbf{w})\rangle \cdot \alpha=\alpha$.

The theorem now follows. 


\section{References}

[1] A. Beilinson, A. Bernstein and P. Deligne, Faisceaux pervers, Astérisque 100 (1982).

[2] A. Borel and J. Moore, Homology theory for locally compact spaces, Michigan Math. J. 7 (1960), 137-159.

[3] N. Chriss and V. Ginzburg, Representation theory and complex geometry, Birkhäuser, Boston, 1997.

[4] W. Crawley-Boevey, Geometry of the moment map for representations of quivers, Compositio Math. 126 (2001), 257-293.

[5] V. G. Kac, Infinite dimensional Lie algebras, 3rd ed., Cambridge Univ. Press, Cambridge, 1990.

[6] A. King, Moduli of representations of finite dimensional algebras, Quart. J. Math. Oxford Ser. (2) 45 (1994), 515-530.

[7] N. Kwon, Borel-Moore homology and K-theory on the Steinberg variety, Michigan Math. J. 58 (2009), 771-781.

[8] G. Lusztig, On quiver varieties, Adv. in Math. 136 (1998), 141-182.

[9] H. Nakajima, Instantons on ALE spaces, quiver varieties and Kac-Moody algebras, Duke Math. J. 76 (1994), 365-416.

[10] H. Nakajima, Quiver varieties and Kac-Moody algebras, Duke Math. J. 91 (1998), 515-560.

[11] P. E. Newstead, Introduction to moduli problems and orbit spaces, Tata Institute Lectures 51, Springer-Verlag, 1978.

[12] D. Yamakawa, Geometry of multiplicative preprojective algebra, IMRP 2008 (2008), 77pp.

Namhee Kwon

Department of Mathematics, Daegu University,

Daegu 712-714, Korea.

E-mail: nkwon@daegu.ac.kr 